\title{
Human-Vehicle Intersection Traffic Lights Timing Optimization Research
}

\author{
Wen Li $\mathbb{D},{ }^{1}$ Hongying Zhang, ${ }^{2}$ Zhaoguo Huang $\mathbb{D}{ }^{2}$ and Chenhui $\mathrm{Li}^{3}$ \\ ${ }^{1}$ School of Automobile, Chang'an University, Xi'an 710064, Shaanxi, China \\ ${ }^{2}$ School of Civil Engineering, Lanzhou University of Technology, Lanzhou 730050, Gansu, China \\ ${ }^{3}$ College of Transportation Engineering, Chang'an University, Xi'an 710064, Shaanxi, China
}

Correspondence should be addressed to Zhaoguo Huang; seuhuang369@hotmail.com

Received 7 December 2021; Revised 22 January 2022; Accepted 8 February 2022; Published 3 March 2022

Academic Editor: Qizhou Hu

Copyright $\left({ }_{0} 2022\right.$ Wen Li et al. This is an open access article distributed under the Creative Commons Attribution License, which permits unrestricted use, distribution, and reproduction in any medium, provided the original work is properly cited.

\begin{abstract}
The purpose of this research is to find a traffic light timing optimization scheme. During the research, an intersection between Xi' an Mingguang road and the Fourth FengCheng road was chosen to analyze the crossing time distribution of pedestrians who were separated from west-to-the-right-turn vehicles during which the method of breaking off both ends of pedestrian green light signals was used. The VISSIM software was used for traffic simulation, aimed at improving traffic volume and right-turning vehicle average speed for less vehicle queuing delays, less human-vehicle conflicts, and better security for pedestrians without excessive interruption on their street crossing efficiency. The optimal scheme is obtained and the result shows that (1) the number of passing vehicles remains unchanged, with the queuing delay reduced by $5.78 \%$ and crosswalk passing speed increased by $19.01 \%$ compared with the original one. (2) As the scheme effect is positively correlated with the increase of right turn vehicle numbers, the scheme could be adopted for urban traffic management based on the local situation, which is not only in peak traffic hours but also in the flat peak time to ensure vehicle efficiency and pedestrian safety in the light of "vehicle yielding to pedestrians" regulation. (3) The scheme could also be adopted in cities with no "vehicle yielding to pedestrians" policy for both people-vehicle separation and pedestrian safety when crossing streets.
\end{abstract}

\section{Introduction}

In recent years, with rapid social and economic development, great progress in urban road infrastructure construction and traffic management, a rapid increase in road traffic demand, and a gradual increase in the supply of transportation infrastructure and service facility pressure, the social requirements of the urban road traffic system are facing intensified contradiction between high and low management levels, along with seriously congested urban traffic, seriously chaotic traffic order, and a decrease in transport efficiency and urban traffic safety.

Various conflicts between pedestrians and vehicles in urban transportation have existed for many years, and the attention paid to pedestrian safety is much less than that to motor vehicle safety concerns with vehicle-vehicle interaction. Being vulnerable to serious injury or even death in road traffic accidents, pedestrians are generally considered as vulnerable road users, and therefore when they are crossing the road, vehicle drivers must give way to pedestrians according to the rule called "vehicle yielding to pedestrians." This policy also requires right-turning vehicles to stop and yield to crossing-road-pedestrians, although there are no traffic signals for right-turning vehicles.

According to traffic regulations in Xi'an, right-turning vehicles at the interaction must yield to pedestrians in zebra crossing area to cross. Such regulation improves traffic volume and pedestrian safety, yet it also causes the queuing and backlog of right-turning vehicles, and even reduces the traffic volume in other directions at single-lane or two-lane junctions, thus aggravating traffic congestion.

It frequently occurs that signal timing at urban road intersections in certain directions cannot meet the actual traffic situation demand or much longer than the actual need 
due to the increase in car ownership. Therefore, intelligent traffic light control systems based on traffic volume [1] and traffic density [2] appear to provide real-time control of traffic lights in order to meet the traffic needs of vehicles at the intersection. Other research on traffic light timing optimization includes studies on travel time [3] and intelligent traffic based on lane [4].

Wang et al. focus on a passing dilemma at $Y$ intersection in order to guarantee the reciprocity of the travelers' efficiency and fairness by proposing an intelligent transportation points system (ITPS) based on the Elo rating system [5].

Meanwhile, video-based intelligent traffic signal light timing optimization is presented in the process of video recognition application in the research [6]. In domestic and foreign research on traffic subject identification in intersection areas, a pedestrian detection technology called YOLO-V3-algorithm-based pedestrian identification research [7] could judge whether there is a pedestrian nearby in a given video and image and mark the location of the pedestrian with the use of computers [8].

In the study on "vehicle yielding to pedestrians," some experts take Beijing for example, and focus on the management strategies of vehicle surrender at crosswalks for the purpose of strengthening the traffic order of vehicles and pedestrians [9].

In the study of pedestrian crossing behavior, in order to analyze conformity in pedestrians' violation of crossing behavior, structural characteristics and evolution rules of pedestrian networks were studied by using the theory of network [10]. At the same time, someone aimed at the factors influencing pedestrians' decisions to cross the road [11]. Wang et al. used the machine vision intelligent algorithm to analyze the yielding behavior of three types of vehicles under the "pedestrian priority" policy [12], moreover, they proposed a dynamic decision model of jaywalking for pedestrians based on the extended decision field theory [13].

Existing traffic signal models focus more on improving traffic efficiency or carrying capacity at intersections or traffic networks and less on pedestrian safety. The goal of this type of model is to divert vehicles, and the goal of the model used in this paper is to balance traffic efficiency and pedestrian safety in the case of large traffic volumes.

At present, there is no research that considers vehicle traffic efficiency while improving pedestrian crossing safety in the policy of "vehicle yielding to pedestrians." This paper is beneficial to pedestrian crossing safety without excessively affecting traffic efficiency.

This paper studies the impact of traffic light timing on right-turning vehicles and the related conflict in the situation of yielding to pedestrians, with pedestrians' crossing motion characteristics analyzed and right-turning vehicle and pedestrian traffic light timing optimized for the balance between pedestrian safety and right-turning vehicle volume, which is of great significance in constructing civilized traffic order and reducing urban vehicle traffic congestion.

It can guide and manage the traffic flow of current urban road traffic more accurately and optimize the timing of traffic lights at intersections during peak urban road traffic. The paper presents a traffic light timing optimization scheme obtained in the study of the traffic problems of right-turning vehicles and pedestrians crossing under vehicle yielding circumstances, during which the intersection of Mingguang Road and Fengcheng Road 4 in Xi'an is taken as an example.

\section{Methods}

2.1. Data Collection. The data in this paper are taken at the intersection of Mingguang Road and Fengcheng Road 4 in Xi' an on April 7, 2021. The survey period is 7:45-8:45 in the morning rush hour (7:00-9:00). The 10-minute video cycle is finally selected between 7:55-8:05 and 8:25-8:35. The two videos of 35 are 20 minutes in total, and the two time periods in the middle of one hour are selected, which can reflect the traffic conditions of motor vehicles and pedestrians in the morning rush hour to some extent. The weather is good and the road surface is dry during the shooting process.

The data obtained include intersection geometry data, motor vehicle traffic volume, pedestrian traffic volume, pedestrian crossing time, and average time as well as phase data of existing signal lights. The data of existing signal lights at the intersection is shown in Figure 1.

2.2. Data Processing. The data are processed by the manual counting method. Finally, the motor vehicle traffic volume and pedestrian traffic volume of two videos each for 20 minutes are averaged and then expanded to an hourly traffic volume. Meanwhile, the pedestrian crossing time is obtained. The pedestrian crossing speed, the distribution of the number of pedestrian crossings over time, and the cumulative distribution of the number of pedestrian crossings are obtained by mathematical operation.

\subsection{Design of Optimization Scheme: "Nip off the Tail"}

2.3.1. Design Idea of Timing Optimization Scheme. In the process of reading video data and simulating the traffic model in chapter 4 , it is found that there are two conflict points between vehicles turning right to the west and pedestrians crossing the street, as shown in Figure 2. The blue rectangular box is the right-turning motor vehicle, and the yellow circular box is the pedestrians crossing the street.

In Figure 2, in the vehicle yielding circumstances, rightturn vehicles must stop to yield the pedestrian cross in any direction. Traffic light signals in the original light timing signals is not continuous ones with about $20 \mathrm{~s}$ of free time, which is too short to be a smooth passage time for right-turn vehicles when vehicles have to spend the rest of the time yielding to pedestrians. Therefore, it not only has a certain impact on the smooth peer of right-turn vehicles but also causes the interruption of right-turning vehicles and increases motor vehicle time and fuel machinery costs as frequent starting and stopping is not conducive to vehicle efficiency and fuel consumption.

According to our optimized scheme, west-bound and south-bound green light signals are connected and made 


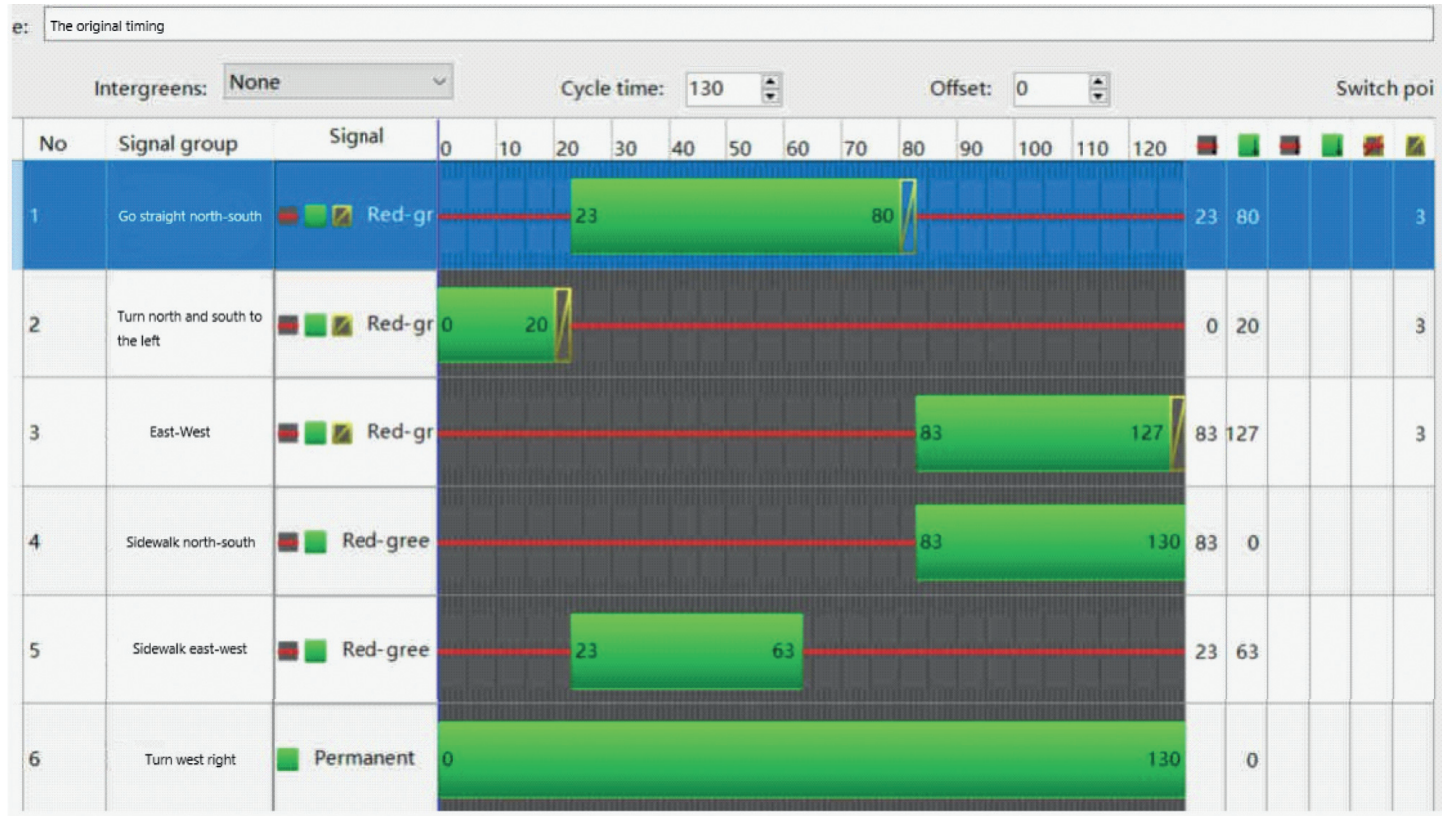

Figure 1: Timing of existing signal lights at the intersection.

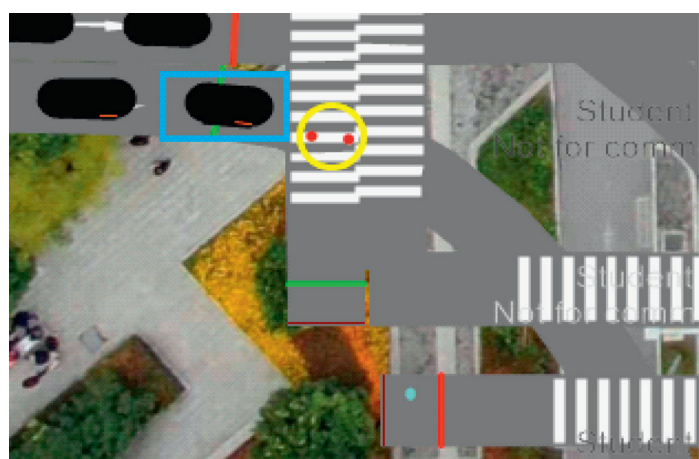

(a)

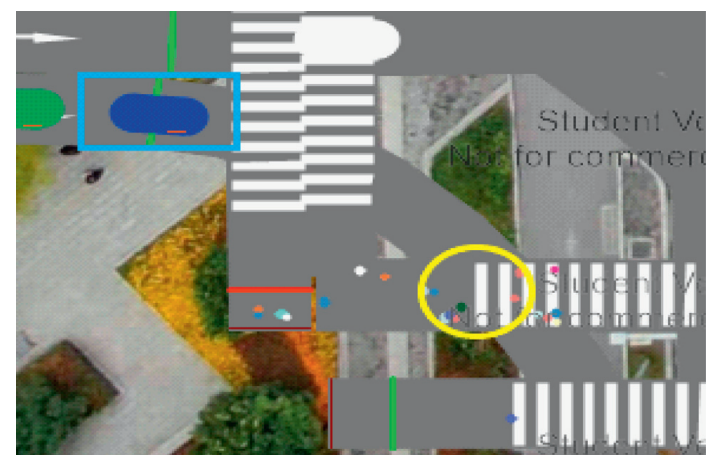

(b)

Figure 2: Collision between vehicles and pedestrians, the blue box represents a vehicle, the yellow box represents pedestrians. (a) The collision between a right-turning vehicle and a westbound pedestrian. (b) The collision between a right-turning vehicle and a southbound pedestrian.

rational reduction by "pinching both ends" based on pedestrians' crossing time distribution, and traffic lights are set for the right-turn lane to separate motor vehicle flow and pedestrian flow for the improvement of traffic volume and speed of right-turn vehicles and the reduction of queuing delays.

Breaking off head means only pedestrian passing time (T1) is reserved, during which the pedestrian arrival rate is close to $90 \%$ or even $100 \%$, and it is enough for most pedestrians crossing the road.

Breaking off tail means to taking advantage of pedestrian crossing time distribution toward a certain direction to provide time enough for most pedestrians crossing the road by setting the time when most pedestrians reach the road $(70 \%-80 \%)$ as pedestrian prohibiting time (T2) before extending the average pedestrian crossing time (T3).
Among them, the formula of timing optimization scheme is as follows:

$$
\mathrm{T}_{-} \text {head }=\mathrm{T} 1,
$$

where T_ head: the green light signal duration of "nip head."

$$
\mathrm{T}_{-} \text {tail }=\mathrm{T} 2+\mathrm{T} 3 \text {, }
$$

where $\mathrm{T}_{-}$tail : the green light signal duration of "de-tail."

\subsubsection{Traffic Light Phase Timing Scheme Design}

(1) "tail removal" for pedestrians crossing the street in the west

When the green signal in the west (south-north) is $18 \mathrm{~s}$, the pedestrian arrival rate is $86 \%$; when the green signal in the west (north-south) is $15 \mathrm{~s}$, the 
pedestrian arrival rate is $88 \%$. The maximum time in the two directions can be used as T2, that is, $T 2=18 \mathrm{~s}$.

The average crossing time of west-facing pedestrians was $T 3=14 \mathrm{~s}$. Therefore, the final westward green signal duration $\mathrm{T}_{-}$tail $=32 \mathrm{~s}$.

(2) "head pinch" for pedestrians crossing the street in the south

The average time of southbound pedestrian crossing is $\mathrm{T}_{-}$head $=23 \mathrm{~s}$.

It is obtained from the original scheme of traffic light signal timing in the intersection, to remove the tail of green traffic light for westbound and the head of green traffic light for the north and south, green traffic light for westbound and north-and-south bound should be connected first, and westbound green traffic light time length is $32 \mathrm{~s}$ and northand-south-bound, $23 \mathrm{~s}$. Concrete intersection traffic signal length are shown in Table 1 and Figure 3.

In the signal control machine of VISSIM, the specific timing scheme of traffic lights at crossroads is shown in Figure 3.

2.4. Evaluation Indexes of the Optimization Scheme. In this paper, the evaluation indexes of the optimization effect of the final scheme are traffic volume, queuing delay, and location speed.

2.5. VISSIM Simulation. In VISSIM, the functions of vehicle and pedestrian input, vehicle path, vehicle signal light, traffic order, and data collector of the simulation model are configured according to the data obtained from the preliminary investigation. In VISSIM, the conflict zone is used to stipulate the traffic rules of "car giving people," and the data collector is set up at the end of the action of the vehicle turning right from west to right, and the simulation model of VISSIM is finally established. The duration of the traffic simulation using VISSIM is 520 seconds. Considering the limitation of the simulation duration and the signal light cycle factor, the simulation duration is selected as a multiple of a complete signal light cycle to reduce the deviation.

The simulation model interface and simulation operation are shown in Figure 4.

\section{Research Results}

3.1. Simulation Results of the Original Scheme. In the process of the simulation of the original scheme, there was only an obvious conflict between "turning west to right" and pedestrians crossing the street southward, and there were vehicles waiting in line. Therefore, traffic light timing optimization is only made to conflict direction.

At the end of the simulation, it is necessary to output the evaluation parameters after the simulation is completed, including traffic volume (the number of vehicles passing), queuing delay, and speed (location speed). The final simulation output results are shown in Table 2.
3.2. Analysis of Pedestrian Crossing Speed. After the pedestrian crossing time is obtained, the pedestrian crossing speed can be calculated according to the pedestrian crossing length $(S)$ and the pedestrian crossing time $(t)$, as shown in the following equation:

$$
V=\frac{S}{t} .
$$

The final pedestrian crossing speed is shown in Table 3.

3.3. Analysis of Pedestrian Crossing Time Distribution. In this section, the calculation method of the time distribution data of pedestrian crossing is shown as follows:

$$
P(k)=\frac{N_{k}}{N}
$$

where $P(k)$ : percentage of pedestrians crossing at the $k$-th second, $N_{k}$ : number of pedestrians crossing in the $k$-th second, and $N$ : total number of pedestrian crossings

$$
P(k)=\frac{N_{k}}{N}
$$

where $P(0 \sim k)$ : percentage of pedestrians crossing in $0 \sim k$ seconds

Excel is used to draw the time distribution data of pedestrian crossings in four directions into a broken line graph, which can intuitively reflect the trend of pedestrian arrival percentage changing with time, as shown in Figure 5.

As can be seen from the above statistics, when the green signal in the west (south-north) is the 18th second, the pedestrian arrival rate is $86 \%$; the pedestrian arrival rate was $88 \%$ when the green signal was 15 seconds west (northsouth); when the green signal in the south (west-east) direction is at the 15 th second, the pedestrian arrival rate is $88 \%$; and when the green light signal in the south (east-west) direction was 16 seconds, the pedestrian arrival rate was $86 \%$. Therefore, in the optimization scheme, the traffic efficiency that does not excessively affect pedestrians can be set as "pedestrian arrival rate greater than $85 \%$."

3.4. Simulation Results of the New Scheme. When the traffic light timing scheme is completed, the simulation program can be run. At the end of the simulation, the evaluation parameters after the simulation should be output, which are traffic volume (number of vehicles passing), queuing delay, and speed (location speed), respectively. The final simulation output results are shown in Table 4 .

As can be seen from Table 4, compared with the original plan, the number of vehicles passing is the same, the queuing delay is reduced by $5.78 \%$, and the speed after crossing the crosswalk is increased by $19.01 \%$. Although the number of vehicles passing through scheme 1 has remained unchanged compared with the original scheme, the queuing delay time of vehicles has been slightly reduced, and the speed of traffic after the crosswalk has been greatly increased. Therefore, it can be shown that the optimization of scheme 1 is effective compared with the original scheme. 
TAble 1: Traffic light signal duration of scheme 1.

\begin{tabular}{|c|c|c|c|c|c|c|}
\hline \multicolumn{7}{|c|}{ Scheme 1} \\
\hline Project & $\begin{array}{c}\text { Phase } 1 \text { north and south } \\
\text { straight (s) }\end{array}$ & $\begin{array}{c}\text { Phase } 2 \text { north and south } \\
\text { left turn (s) }\end{array}$ & $\begin{array}{c}\text { Phase } 3 \text { east and } \\
\text { west (s) }\end{array}$ & $\begin{array}{c}\text { Phase } 4 \text { north and south } \\
\text { sidewalks (s) }\end{array}$ & $\begin{array}{c}\text { Phase } 5 \text { east-west } \\
\text { sidewalk (s) }\end{array}$ & $\begin{array}{l}\text { Phase } 6 \\
\text { right turn }\end{array}$ \\
\hline Total cycle & 130 & 130 & 130 & 130 & 130 & 130 \\
\hline Red & 67 & 107 & 83 & 80 & 96 & 55 \\
\hline Yellow & 3 & 3 & 3 & 0 & 0 & 0 \\
\hline Green & 60 & 20 & 44 & 23 & 32 & 75 \\
\hline
\end{tabular}

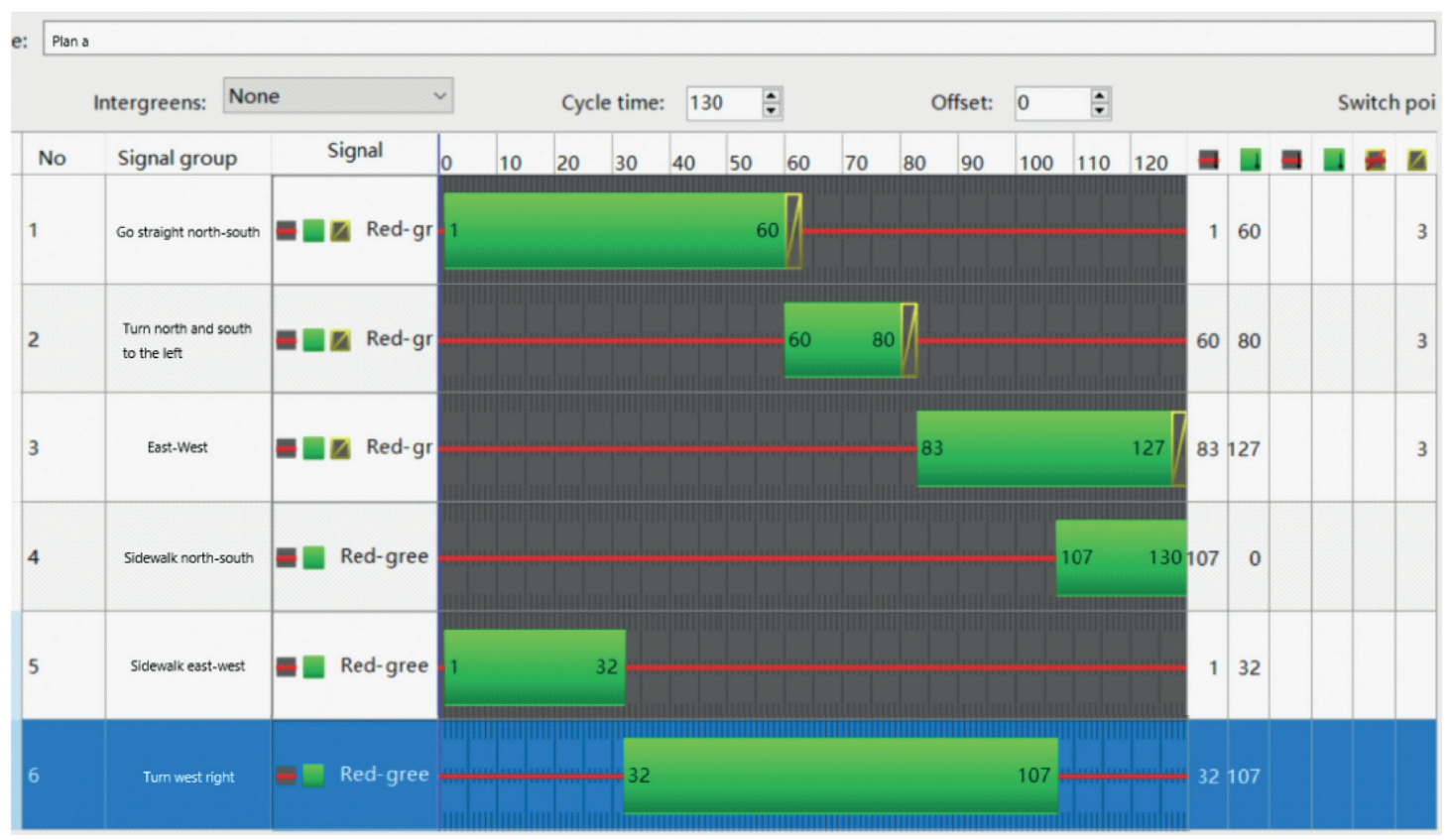

FIGURE 3: The signal control machine of VISSIM.

3.5. Discussion. The paper puts forward a method of "breaking off both ends" to make right-turn vehicle and pedestrian crossing traffic light timing optimization which is obtained by comparing traffic light timing scheme of the right-turn vehicle and pedestrian crossing road at the moment of conflict between the two sides in a particular intersection, analyzing pedestrian -crossing- road time distribution to the certain direction before getting time scan of traffic green light signal for pedestrian. After that, VISSIM software was used to establish and simulate the traffic model of the original scheme and scheme one. The data acquisition device was used to output the results of three indicators of traffic condition evaluation: vehicle traffic volume, queuing delay, and speed. The comparison results were obtained and the optimization of scheme one was confirmed to be effective compared with the original scheme.

However, in the scheme of a simulation evaluation, an obvious increase only occurs in the index of "traffic speed." There is no significant increase in the other two indexes, such as "traffic volume" and "queuing delay." The causes are summed up by comparing the data with the real-time status during the simulation and stated as follows:

(1) The simulation time is not long enough, and the random generation of vehicles may affect the simulation results and cause errors.
(2) The number of right-turning vehicles in the original data is not enough, resulting in no significant optimization effect compared with the original scheme in terms of the traffic volume and queuing delay of right-turning vehicles.

3.5.1. Prolonging the Simulation Optimization Time. In order to discuss the influence of simulation duration on optimization results, the model was simulated after obtaining the new version of the software, and the output results of scheme 1 and the original scheme were compared, as shown in Table 5.

According to the comparison in Table 5, compared with the original scheme, the number of vehicles passing through after the extension of simulation time increases slightly, while the queuing delay decreases by $30.29 \%$ and the speed increases by $15.99 \%$. Compared with the original simulation time, the optimization effect is more obvious.

3.5.2. Expansion of Traffic Volume. In order to discuss whether the number of vehicles turning right will affect the scheme before and after optimization, the measure adopted here is to expand the traffic volume of vehicles turning right 

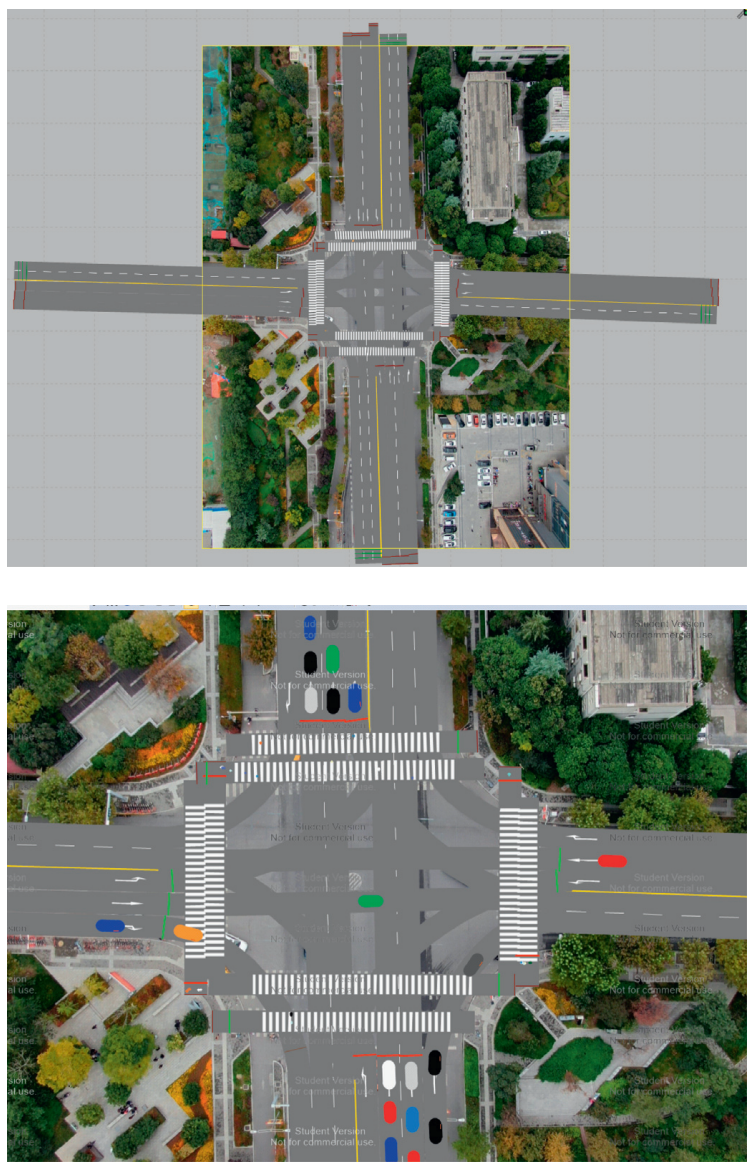

FIgURE 4: Simulation interface and operation diagram.

TABLE 2: Simulation results of the original scheme.

\begin{tabular}{lccc}
\hline Simulation time & $\begin{array}{c}\text { Vehicle pass } \\
\text { number }\end{array}$ & $\begin{array}{c}\text { Queuing delay } \\
(\mathrm{s})\end{array}$ & $\begin{array}{c}\text { Speed } \\
(\mathrm{km} / \mathrm{h})\end{array}$ \\
\hline $0-520$ & 63 & 6.77 & 47.65
\end{tabular}

west by one to six times, namely from 450 vehicles/hour to $900,1350,1800,2250,2700$, and 3150 vehicles/hour, respectively. Through the simulation output of VISSIM and the comparison between the optimization scheme and the original scheme, the optimization degree percentage of "number of vehicles through," "queuing delay," and "speed" of different traffic volumes turning from west to right is compared, as shown in Table 6.

In order to better obtain the trend of optimization results, the drawing function in Excel was used to obtain the trend graph of optimization degree, as shown in Figure 6.

It can be seen from Figure 6 that the optimization effect of scheme 1 increases with the increase of the number of right-turning vehicles, especially in the index of "number of vehicles" and "queuing delay," which has a great range of optimization. At the same time, the optimization of the index of "queuing delay" reached its threshold value when the number of right-turning cars reached 1350 cars/hour. After that, no matter how the number of right-turning cars expanded, the optimization effect had little difference with
Table 3: Pedestrian crossing speed statistics.

\begin{tabular}{lcc}
\hline Speed $(\mathrm{km} / \mathrm{h})$ & Quantity & Proportion $(\%)$ \\
\hline Pedestrian crossing speed (south to north) & \\
8.4 & 1 & 1.67 \\
7.4 & 1 & 1.67 \\
6.6 & 3 & 5.00 \\
6.3 & 2 & 3.33 \\
6.0 & 4 & 6.67 \\
5.7 & 9 & 15.00 \\
5.5 & 14 & 23.33 \\
5.3 & 12 & 20.00 \\
5.0 & 7 & 11.67 \\
4.7 & 5 & 8.33 \\
4.5 & 2 & 3.33 \\
\hline Pedestrian crossing speed (north to south) & \\
8.0 & 2 & 5.41 \\
6.5 & 6 & 16.22 \\
6.0 & 2 & 5.41 \\
5.5 & 12 & 32.43 \\
5.1 & 2 & 5.41 \\
4.8 & 5 & 13.51 \\
4.5 & 4 & 10.81 \\
4.2 & 2 & 5.41 \\
3.6 & 2 & 5.41 \\
\hline
\end{tabular}

the threshold value. The optimization of the "number of vehicles" reached its peak when the number of right-turning vehicles reached 1800. After that, although the number of 

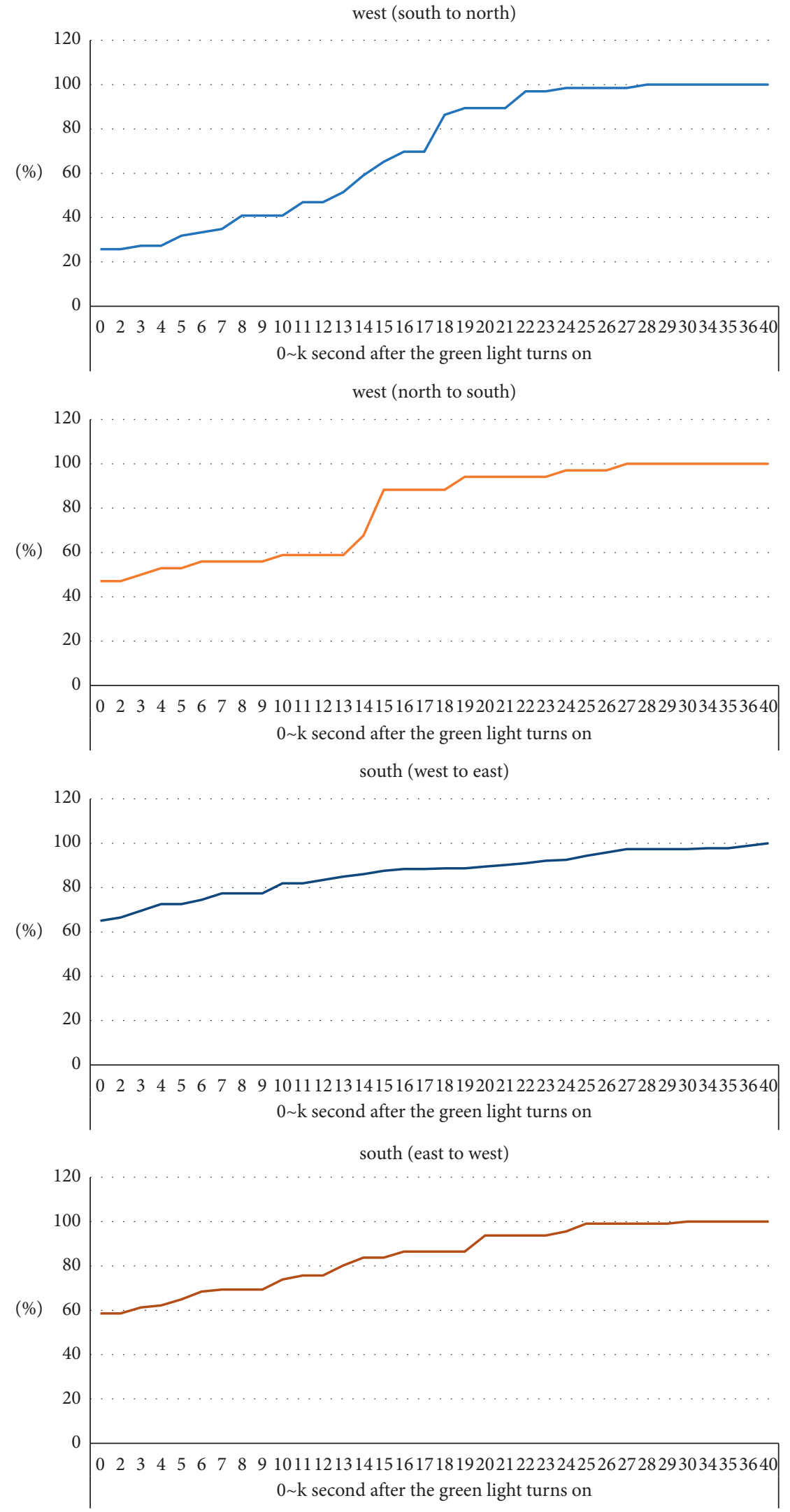

FigURE 5: Time distribution of pedestrians in four directions ( $Y$-axis: the proportion of pedestrians crossing the street). 
TABLE 4: Comparison between scheme 1 and the original scheme.

\begin{tabular}{lccc}
\hline & Number of vehicles through & Queuing delay $(\mathrm{s})$ & \\
\hline Original scheme & 63 & 6.77 & Speed $(\mathrm{km} / \mathrm{h})$ \\
Scheme 1 & 63 & 6.40 & 47.65 \\
\hline
\end{tabular}

TABLE 5: Comparison of the output results of the original scheme and scheme 1 after the extension of simulation time.

\begin{tabular}{lcccc}
\hline Scheme & Simulation time & Number of vehicles & Queuing delay $(\mathrm{s})$ & Speed $(\mathrm{km} / \mathrm{h})$ \\
\hline Original scheme & $0-5200$ & 649 & 17.1 & 49.29 \\
Scheme 1 & $0-5200$ & 653 & 11.92 & 57.17 \\
\hline
\end{tabular}

TAвLE 6: Optimization degree of the original scheme with different number of vehicles turning from west to right.

\begin{tabular}{|c|c|c|c|}
\hline Number of right turn vehicles (vehicles/hour) & Number of vehicles through (\%) & Queuing delay (\%) & Speed (\%) \\
\hline 450 & 0 & 5.78 & 19.01 \\
\hline 900 & 20.59 & 56.43 & 19.33 \\
\hline 1350 & 69.45 & 67.86 & 18.87 \\
\hline 1800 & 106.86 & 68.43 & 15.51 \\
\hline 2250 & 101.92 & 66.61 & 17.70 \\
\hline 2700 & 100.95 & 66.75 & 17.46 \\
\hline 3150 & 91.82 & 64.81 & 15.91 \\
\hline
\end{tabular}

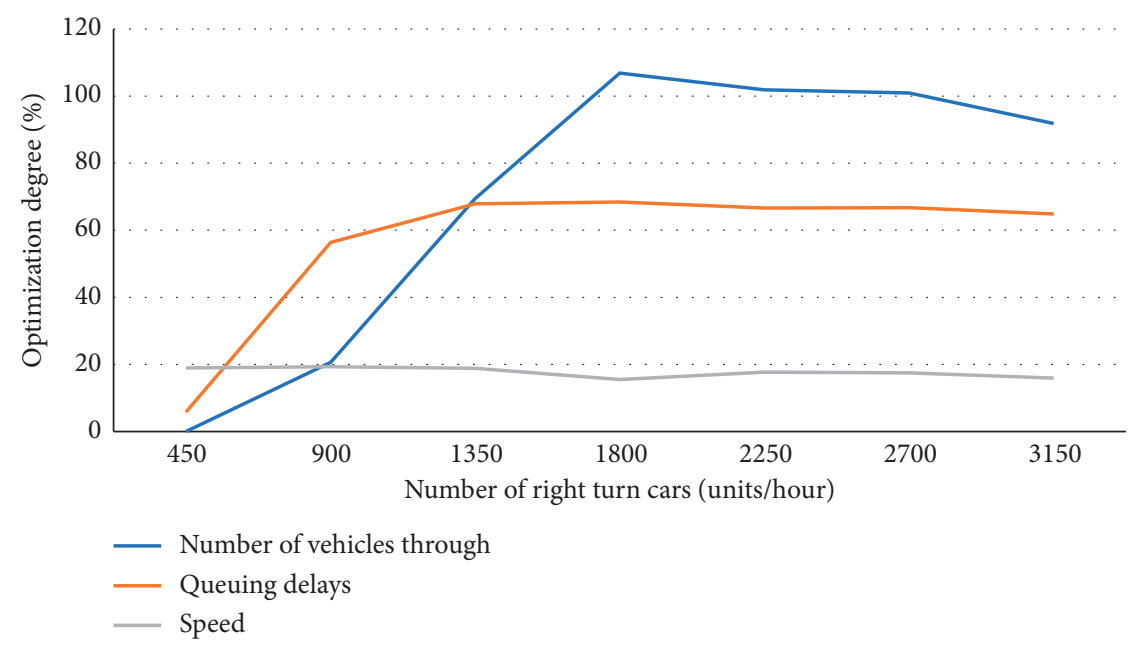

FIGURE 6: Influence of the number of right-turning vehicles on the optimization effect of scheme 1.

right-turning vehicles increased, the number of vehicles passing during the simulation period decreased.

\section{Conclusion}

In this paper, "breaking both ends" is used to make traffic light signal optimization for right-turn vehicles and crossing-road-pedestrians during which the comparison between two traffic signal timing distribution schemes for rightturning vehicles to a certain direction and road-crossing pedestrians that is meeting conflict with vehicles in a certain intersection before determining time length of green traffic light signal timing scheme for crossing road pedestrian and optimization scheme is obtained. After that, VISSIM software was used to establish and simulate the traffic model of the original scheme and scheme one. The data acquisition device was used to output the results of three indicators of traffic condition evaluation: vehicle traffic volume, queuing delay, and speed. The comparison results were obtained and the optimization of scheme one was confirmed to be effective compared with the original scheme.

The method used in this paper should be appropriate according to the situation. Therefore, the recommendations are as follows:

(1) It should be used at intersections with large traffic volumes.

(2) It can be used at the intersection during rush hours so as to better play the optimization role of the method. 


\section{Data Availability}

The data used to support the findings of this study are included within the article.

\section{Conflicts of Interest}

The authors declare that they have no conflicts of interest.

\section{References}

[1] D. Wang and M. Li, "AN intelligent traffic lights control algorithm based ON traffic flow," Computer Applications and Software, vol. 32, no. 1, pp. 241-244, 2015.

[2] B. Pratama, J. Christanto, M. T. Hadyantama, and A. Muis, "Adaptive traffic lights through traffic density calculation on road pattern," in 2018 International Conference on Applied Science and Technology (iCAST), Manado, Indonesia, 2018.

[3] D. Helbing, "A section-based queueing-theoretical traffic model for congestion and travel time analysis in networks," Journal of Physics, vol. 36, pp. L593-L598, 2003, (Pubitemid 37470014).

[4] H. Prothmann, J. Branke, H. Schmeck et al., "Organic traffic light control for urban road networks," International Journal of Autonomous and Adaptive Communications Systems, vol. 2, no. 3, pp. 203-225, 2009.

[5] Y. Wang, Z. Yao, C. Wang, J. Ren, and C. Qiao, "The impact of intelligent transportation points system based on Elo rating on emergence of cooperation at Y intersection," Applied Mathematics and Computation, vol. 370, p. 124923, 2020.

[6] A. Kanungo, A. Sharma, and C. Singla, "Smart traffic lights switching and traffic density calculation using video processing," 2014 Recent Advances in Engineering and Computational Sciences (RAECS), pp. 1-6, 2014.

[7] W. Li, Infrared Image Pedestrian Detection via YOLO-V3, , pp. 1052-1055, IEEE 5th Advanced Information Technology, Electronic and Automation Control Conference (IAEAC), 2021.

[8] A. Menon, B. Omman, and A. S, "Pedestrian counting using yolo V3," International Conference on Innovative Trends in Information Technology (ICITIIT), pp. 1-9, 2021.

[9] H. JiangDi Huang et al., "On the effect of interference caused by mixed traffic at signalized intersection[J]," Journal of Transportation Systems Engineering and Information Technology, vol. 6, no. 3, pp. 75-78, 2006.

[10] Z.-ping Zhou, W.R Wang, and Li GangLu, "Modeling pedestrian's conformity violation behavior based on networks analysis[J]," Journal of Beijing University of Technology, vol. 40, no. 3, pp. 374-377, 2014.

[11] N. Mahmud Zafri, R. Sultana, and M. d, "Rakibul Hasan Himal, Tanzila Tabassum, Factors influencing pedestrians' decision to cross the road by risky rolling gap crossing strategy at intersections in Dhaka, Bangladesh," Accident Analysis \& Prevention, vol. 142, 2020.

[12] Y. Wang, Q. Su, C. Wang, and C. Prato, "Investigating yielding behavior of heterogeneous vehicles at a semi-controlled crosswalk," Accident Analysis \& Prevention, vol. 161, p. 106381, 2021.

[13] Y. Wang, B. Shen, H. Wu, C. Wang, Q. Su, and W. Chen, "Modeling illegal pedestrian crossing behaviors at unmarked mid-block roadway based on extended decision field theory," Physica A: Statistical Mechanics and its Applications, vol. 562, p. $125327,2021$. 